\title{
Growing Hierarchical Self-Organizing Map for Alarm Filtering in Network Intrusion Detection Systems
}

\author{
Ahmad FAOUR \\ INSA of ROUEN \\ Laboratoire LITIS \\ Email: ahmad.faour@ul.edu.lb
}

\author{
Philippe Leray \\ INSA of ROUEN \\ Laboratoire LITIS \\ Email: pleray@insa-rouen.fr
}

\author{
Bassam Eter \\ Lebanese University \\ Laboratoire LPM \\ Email: beter@ul.edu.lb
}

\begin{abstract}
It is a well-known problem that intrusion detection systems overload their human operators by triggering thousands of alarms per day. This paper presents a new approach for handling intrusion detection alarms more efficiently. Neural Network analyses based on the self-organizing map (SOM) and the growing hierarchical self-organizing map (GHSOM) are used to discover interrest patterns signs of potential scenarios of attacks aiming each machine in the network. The GHSOM addresses two main limits of SOM which are caused, on the one hand, by the static architecture of this model, as well as, on the other hand, by the limited capabilities for the representation of hierarchical relations of the data. The experiments conducted on several logs extracted from the SNORT NIDS, confirm that the GHSOM can form an adaptive architecture, which grows in size and depth during its training process, thus to unfold the hierarchical structure of the analyzed logs of alerts.
\end{abstract}

\section{INTRODUCTION}

With the ever growing deployment of networks and the Internet, the importance of network security has increased. Over the past ten years, the number as well as the severity of network-based computer attacks have significantly increased [1]. As a consequence, classic computer security technologies such as authentication and cryptography have gained in importance. Simultaneously, intrusion detection has emerged as a new and potent approach to protect computer systems [2], [3]. In this approach, so-called Intrusion Detection Systems $(I D S s)$ are used to monitor and analyze the events occurring in a computer system. Recently, we have witnessed many theoretical and semi-practical approach to use data mining technology in the area of intrusion detection [4]. The governing approach was to use data mining algorithms to detect anomalies that represent intrusions, or attacks. There was an attempt to use data mining as a replacement for existing intrusion detection techniques. However, except for specific areas there is no practical implementation of this approach. The main reason being that existing technologies suffice for detecting intrusions, and, that data mining does not provide enough context to serve as an intrusion detection tool. Regardless of the development in the Security Information area, there is still a huge problem with existing tools. For both signature based and behavioral rules approach, most of the rules are too generic. The consequence is that although these tools detect all intrusions, they detect much more than that. This problem, known as false positives, is a big barrier for intrusion detection tools to cross before their deployment can be practical. To date, security experts, are struggling with an inherent conflict and are sometimes forced to write less adequate detection rules just to reduce the number of false positives. In this paper we suggest a different approach for using data mining technology in the intrusion detection area. We claim that the best positioning for a data mining technology within an intrusion detection system is not as a detection engine, but rather as an analysis layer that will filter out the false positives. In our past researches [?], [?], we proposed an automatic process that consists of two steps. The first step aims at grouping similar standard behavior for external machines with destination to internal machines using clustering algorithms like self-organizing maps [5]. The second step uses these information for determining if the network is really attacked and filter out the false positives using probabilistic tools like Bayesian Networks [6]. The SOM has shown to be exceptionnally successful in arranging high-dimensional input data along its two-dimensional output space such that similar inputs are mapped onto neighboring regions of the map. In other words, the similarity of the input data is preserved as faithfully as possible within the representation space of the SOM. Despite the large number of research reports on application of the SOM [?] some deficiencies remained largely untouched. First, the SOM uses a static network architecture both in terms of number and arrangement of neural processing elements, which have to be defined prior to the start of training. Second, hierarchical relations between the input data are rather difficult to detect in the map display. So far, both issues are have been addressed seperately by means of adaptive architectures, e.g. the Growing Grid [?], or hierarchies of independent SOMs, e.g. the Hierarchical Feature Map [?] or the Tree-structured SOM[?].

In this paper, we propose a neural network model, namely the Growing Hierarchical Self-Organizing Map (GHSOM)[7], that addresses both deficiencies as outlined above within one framework. Basically, this neural network model is composed 
of independent SOMs, each of which is allowed to grow in size during the training process until a quality criterion regarding data representation is met. This growth process is further continued to form a layered architecture such that hierarchical relations between input data are further detailed at lower layers of the neural network.

The outline of this paper is as follows. Section 2 gives an overview of the limitation of the intrusion detection systems and discusses related works. Section 3 gives a breif overview of our approach proposed in [?]. in which we used SOM, and compare it by GHSOM. Section 4 shows the experiments and the obtained results and finally section 5 concludes and presents our future works and perspectives.

\section{Network InTRUsion Detection Systems}

The NIDS (Network Intrusion Detection System), software created to detect the abnormal activities on the network, generally function by signatures, on the same principle of anti-virus softwares [8]. The known attacks are indexed in signature databases and each time that an activity resembles one of these attacks, an alarm is generated. When a new exploit (successful intrusion attempt) is detected, an adapted signature will be added to the signature database. Another approach, sometimes used jointly with the preceding one, is a statistical approach. During a training phase (i.e., adjustment of the parameters of the statistical model), the NIDS learns a standard profile (number of exchanged packets, volume of flow, numbers of connections, etc...) from the network and alarms the administrator when the traffic deviates this profile.

The main problem that the administrators discover when they install a NIDS is the huge amount of generated alarms (false positives). On an average size network (one or two public classes C), several thousands of alarms are generated daily making almost impossible the analysis of the results. The consequence is that the administrator has to seriously increase his tolerance level, which will lead it to miss many real problems and to make it possible to an advanced intruder to make successfully of a sufficiently discrete attack not to be detected a whole. In fact, the NIDS is not able to judge relevance, gravity and correlation of the alarms. It generates so much alarms which it will be very difficult to detect the serious problems between all alarms.

\section{A. Related work}

The intuitively most appealing way of dealing with false positives is to build "better" IDSs, which trigger less false positives. This is a challenging endeavor because false positives are the result of multiple problems. Examples of IDSs that are less prone to false positives include the embedded detectors technology by [9], a lightweight tool for detecting Web server attacks by [10], and a network-based IDS that focuses exclusively on low-level network attacks [11].

Recently, there have been several proposed techniques for filtering the alarms issued by the NIDS. All these techinques used post-processing methods that uses the alarms as input and try to give the administrator the dangerous alarms and filter

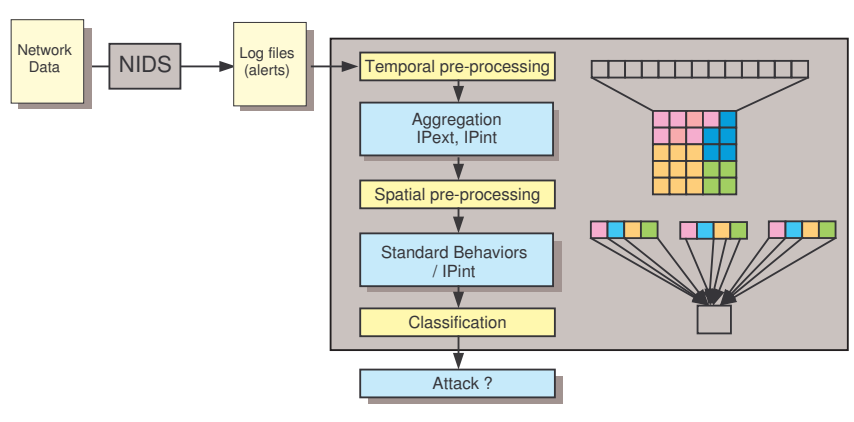

Fig. 1. General description of our approach

out the rest. Alarm correlation systems [12], [?], [13], [14], [?] try to group alarms so that the alarms of the same group pertain to the same type (e.g., the same attack). In that way, they offer a more condensed view on the security issues raised by an IDS [15]. These techniques can be classified in three general categories: (a) Alert Clustering ([?], [?]): they use probabilistic-based reasoning to correlate alerts by measuring and evaluating the similarities of alert attributes. (b) Matching predefined attack scenarios: Debar and Wespi [13] apply backward and forward reasoning techniques to correlate alerts with duplicate and consequence relationship. Morin and Debar [16] apply chronicle formalism to aggregate and correlate alerts. The approach performs attack scenario pattern recognition based on known malicious event sequences. The main limitation of this approach is that it rely on various forms of predefined knowledge of attack conditions and consequences. They cannot recognize a correlation when attack is new. (c) Prerequisties / consequences ([17], [18], [19], [20], [20]): They build alert correlation systems based on matching the pre/post-conditions of individual alerts. The idea of this approach is that prior attack steps prepare for later ones. Therefore, the consequences of earlier attacks correspond to the prerequisites of later attacks. The correlation engine searches alert pairs that have a consequence and prerequisite matching. One challenge to this approach is that a new attack cannot be paired with any other attacks because its prerequisites and consequences are not defined.

\section{OUR APPROACH}

The goal of our system is starting from the alarms generated by a NIDS, and filtering these alarms to determine if there were an attack on the network during a fixed lapse of time. Our system is composed of three components showed in figure 1 and detailed in [?].

By considering that a scenario of attack consists of a serie of events proceeding in an interval of time, we start by making a synthesis of alarms generated by the NIDS in a fixed temporal window. This synthesis gives us a summary of the behavior of all the external machines $I P_{\text {ext }}$ (potentially attacking) to all the internal machines $I P_{\text {int }}$ (potentially attacked). The logs used in our experiments contain 406 different types of alerts generated by SNORT [21]. At the end of this phase, we obtain 
a summary [window IP $P_{\text {ext }}$ IP $_{\text {int }}$ Nalert $_{1} \ldots$ Nalert $_{406}$ ] of the behavior of each internal machine in our network gathered for each external machine in connection. To take account of the average traffic of each internal machine we proposed to normalize the data.

In the second part (special prerocessing), we work starting from the number of alarms of each type generated for each couple $\left(I P_{\text {ext }}, I P_{\text {int }}\right)$, by supposing that this vector is a representative behavior of each $I P_{\text {ext }}$ in connection to each $I P_{i n t}$. On the basis of the principle that this behavior can be similar for several external machines connecting to one or more internal machines, we will use a technique of unsupervised classification to cluster these behaviors in a given number of behavior-types. We used a self-organizing map [5] as clustering algorithm. The quality of clustering using SOM are mainly influenced by three essential parameters: (1) size of the map, (2) initialization of weights (codebook vectors) and (3) neighborhood functions.

However, the use of SOM requires to define a priori these three parameters of the map. For choosing the best parameters several validation measures are proposed [22], among which we used the Davies-Bouldin index $(D B)$. The $D B$ index will have a small value for a good clustering. After the choice of the best SOM, we proceed according to two approaches. In the first approach (Approach1), we made a synthesis [window IP $P_{\text {int }}$ dist2clust $_{1} \ldots$ dist clust $_{n}$ ] of the sum of the distance for every vector $\left(I P_{\text {ext }}, I P_{\text {int }}\right)$ to the behavior-types $\left(\right.$ clust $_{1} \ldots$ clust $\left._{n}\right)$ detected for each $I P_{\text {int }}$ independently of $I P_{\text {ext }}$ in a mobile window of time. In the second approach (Approach2), for every $I P_{i n t}$, we made a synthesis of the number of behavior-types detected bound for this $I P_{i n t}$ in a temporal window. By this way, we obtain a

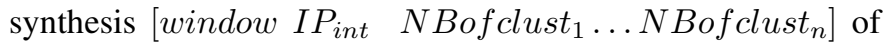
the behavior-types $\left(\right.$ clust $_{1} \ldots$ clust $\left._{n}\right)$ detected for each $I P_{\text {int }}$ independently of $I P_{\text {ext }}$ to be able to compare the profile of two different internal machines.

The synthesis of the behavior-types calculated for every $I P_{\text {int }}$ is supposed to be representative of the various types of potential attacks aiming each internal machine of the network during a fixed window of time. We propose in the last phase (clustering) to use these information to determine if the network were really attacked ( $A T T=$ true or false). To implement this task of classification, we tested two supervised classifiers; the Bayesian networks [23], [24] and the SVM [?].

\section{The Growing Hierarchical SElF-Organizing MAP}

The Growing Hierarchical Self-Organizing Map (GHSOM) [?], which is an extension to the growing grid SOM [?] and hierarchical SOM [?], can build a hierarchy of multiple layers where each layer consists of several independent growing SOMs. The size of these SOMs and the depth of the hierarchy are determined during its learning process according to the requirements of the input data. As depicted in Fig. 2, the GHSOM architecture is similar to a tree structure where the $\operatorname{SOM}(\mathrm{s})$ at each layer can branch out to additional SOMs at the
Basic steps for horizental growth:

1) Initialize the weight vector of each neuron with random values.

2) Perform the traditional SOM learning algorithm for a fixed number $\lambda$ of times.

3) Find the error unit $e$ and its most dissimilar neighbor unit $d$. (Note that the error unit $e$ is the neuron with the largest deviation between its weight vector and the input vectors it represents.)

4) Insert a new row or a new column between $e$ and $d$. The weight vectors of these new neurons are initialized as the average of their neighbors.

5) Repeat steps $2-4$ until the mean quantization error of the map $M Q E_{m}<\tau_{1} * q e_{u}$ where $q e_{u}$, is the quantization error of the neuron $u$ in the preceding layer of the hierarchy.

Basic steps of hierarchical growth:

1) Check each neuron to find out if its $q e_{i}>\tau_{2} * q e_{0}$, where $q e_{0}$ is the quantization error of the single neuron of Layer 0 , then assign a new SOM at a subsequent layer of the hierarchy.

2) Train the SOM with input vectors mapped to this neuron.

TABLE I

Basic steps of the horizontal growth and the hierarchical growth of the GHSOM.

subsequent layer. The upper layers show a coarse organization of the major clusters in the data, whereas the lower layers offer a more detailed view of the data.

For the initial setup of the GHSOM, at Layer 0, a singleneuron SOM is created and the neuron's weight vector is initialized as the average of all input vectors. Then, the learning process starts at Layer 1 with a small SOM (usually a 22 grid) whose weight vectors are initialized to random values.

The GHSOM grows in two dimensions: horizontally (by increasing the size of each SOM) and hierarchically (by increasing the number of layers). For horizontal growth, each SOM modifies itself in a systematic way very similar to the growing grid [12] so that each neuron does not represent too large an input space. For hierarchical growth, the principle is to periodically check whether the lowest layer SOMs have achieved sufficient coverage for the underlying input data. The basic steps of the horizontal growth and the hierarchical growth of the GHSOM are summarized in Table I.

The growth process of the GHSOM is controlled by the following four important factors.

- The quantization error of a neuron $i, q e_{i}$, is calculated as the sum of the distance between the weight vector of neuron $i$ and the input vectors mapped onto this neuron.

- The mean quantization error of the map $\left(M Q E_{m}\right)$ is the mean of all neurons' quantization errors in the map.

- The threshold $\tau_{1}$ is for specifying the desired level of detail that is to be shown in a particular SOM.

- The threshold $\tau_{2}$ is for specifying the desired quality of input data representation at the end of the learning process.

\section{BEHAVIOR TYPES DISCOVERY BY THE GHSOM}

In order to exemplify some of the differences between the SOM and the GHSOM clustering models, the unsupervised classification problem described in Section III is retaken. 
We work starting from the number of alarms of each type generated for each couple $\left(I P_{\text {ext }}, I P_{\text {int }}\right)$ in the temporal preprocessing phase, by supposing that this vector is a representative behavior of each $I P_{\text {ext }}$ in connection to each $I P_{\text {int }}$. Starting from the principle that this behavior can be similar for several external machines connecting to one or more internal machines, we used GHSOM for clustering these behaviors into a number of behavior-types. The number of behavior-types is not defined a priori like the case of SOM, but determined dynamically during the process of the creation of the map. As it is well known, the resulting map is governed by the two parameters $\left(\tau_{1}\right.$ and $\left.\tau_{2}\right)$. Generally, the values for $\tau_{1}$ and $\tau_{2}$ are chosen such that $0<\tau_{2}<<\tau_{1}<1$. The choice of the parameter $\tau_{2}$ is not critical as it is possible to set it to values smaller than necessary and to decide after the training process how many layers are sufficient. On the other hand, $\tau_{1}$ leads to decisions which cannot be undone at later stages without retraining the GHSOM. Setting $\tau_{1}$ to small values will lead to small maps and a deep hierarchy, while large values will result in large maps with a flat hierarchical structure. Usually the user will train and manually evaluate several maps until an appropriate value for $\tau_{1}$ is estimated.

For the interpretation of the obtained maps, we admit the following suppositions:

- the codebook vector of each cluster is a representative of the data projected in this cluster.

- for each codebook vector we determine the most significant variables (for instance, the first top 5 variables). As each variable corresponds to one specific alarm, we obtain the characteristic alarms of each cluster.

- every data projected in a cluster is classified as the more frequent situation also projected in the same location during the learning phase.

\section{EXPERIMENTS AND RESULTS}

\section{A. Description of the Data}

We work starting from log files issued from SNORT NIDS. These files contain 32031 alarms generated over a duration of 20 days from 20/11/2004 to 10/12/2004. These alarms correspond to 4638 external machines trying to connect 288 internal machines. Alarms generated during these 20 days are of 406 different types and include 16 real attacks scenarios, some are of a few minutes, others lasting several days and the other are for normal scenario. The scenarios of attack are distributed as 4 scenarios brute force on POP3, 3 scenarios crawler Web, 2 scenarios Web IIS, 2 scenarios scanner of vulnerability, 1 scenario IIS attack against apache server, 3 scenarios brute force against FTP server and 1 scenario SNMP attack. After implementing the temporal pre-processing phase we decomposed the base in one learning base and one testing base. The learning base contains 10 scenarios of attacks and the testing base contains six scenarios of attacks.

To choose the best couple of the values of $\left(\tau_{1}, \tau_{2}\right)$, we launched a set of experiments varying these two parameters and calculating the percentage of detection of the attacks (detection rate) and the percentage of the "false positive".

\section{B. Quantitative Analysis}

1) Horizental Expansion: We started by fixing the parameter $\tau_{2}=0.03$, and varying the parameter $\tau_{1}$ between $0.4>\tau_{1}>0.1$ to study the influence of the horizontal expansion of the map. The results obtained for the application of the GHSOM on the learning data and validation data are presented in the tables II and III.

TABLE II

THE OBTAINED RESULTS FOR $\tau_{2}=0.03$ ET $0.4>\tau_{1}>0.1$ FOR THE LEARNING DATA.

\begin{tabular}{cccc}
\hline \hline$\tau_{1}$ & $\tau_{2}$ & Detection Rate & False Positive \\
\hline 0.4 & 0.03 & $88 \%$ & $6.56 \%$ \\
0.3 & 0.03 & $95.2 \%$ & $7.3 \%$ \\
0.2 & 0.03 & $95.2 \%$ & $13 \%$ \\
0.1 & 0.03 & $95.2 \%$ & $13 \%$ \\
\hline \hline
\end{tabular}

TABLE III

THE OBTAINED RESULTS FOR $\tau_{2}=0.03$ ET $0.4>\tau_{1}>0.1$ FOR THE VALIDATION DATA.

\begin{tabular}{cccc}
\hline \hline$\tau_{1}$ & $\tau_{2}$ & Detection Rate & False Positive \\
\hline 0.4 & 0.03 & $88 \%$ & $10 \%$ \\
0.3 & 0.03 & $96 \%$ & $8.4 \%$ \\
0.2 & 0.03 & $96 \%$ & $8.7 \%$ \\
0.1 & 0.03 & $96 \%$ & $8.7 \%$ \\
\hline \hline
\end{tabular}

2) Hierarchical Expansion: To study the influence of the vertical expansion (hierarchical) of the map in the representation of the structure of the data, we fix this time $\tau_{1}=0.3$ and we vary $\tau_{2}$ between 0.01 and 0.03 . The smallest is $\tau_{2}$, the deeper will be the hierarchy. The results obtained are presented in the tables IV and V. The best the result is obtained for the couple of value $\left(\tau_{1}=0.3, \tau_{2}=0.01\right)$.

TABLE IV

THE RESULTS OBTAINED FOR $\tau_{1}=0.3$ AND $0.03>\tau_{2}>0.01$ IN THE LEARNING DATA.

\begin{tabular}{cccc}
\hline \hline$\tau_{1}$ & $\tau_{2}$ & Detection Rate & False Positive \\
\hline 0.3 & 0.03 & $95.2 \%$ & $7.3 \%$ \\
0.3 & 0.02 & $96.4 \%$ & $7.38 \%$ \\
0.3 & 0.01 & $96.4 \%$ & $4 \%$ \\
\hline \hline
\end{tabular}

TABLE V

THE RESULTS OBTAINED FOR $\tau_{1}=0.3$ AND $0.03>\tau_{2}>0.01$ IN THE VALIDATION DATA.

\begin{tabular}{cccc}
\hline \hline$\tau_{1}$ & $\tau_{2}$ & Detection Rate & False Positive \\
\hline 0.3 & 0.03 & $96 \%$ & $8.4 \%$ \\
0.3 & 0.02 & $96 \%$ & $4.7 \%$ \\
0.3 & 0.01 & $96 \%$ & $4.7 \%$ \\
\hline \hline
\end{tabular}

From the obtained results, we can notice the influence of the refinement of the map by the degradation of the value of the parameter $\tau_{2}$. Indeed, for $\tau_{2}=0.03$, the obtained map consists of only one level map with 132 clusters. The "false negative" was equal to $4.8 \%$ and the "false positive" 
was equal to $7.3 \%$. The cluster (18) classified as "normal" contains $1.2 \%$ of the vectors belonging to the scenario of attack 9. These vectors are drowned between normal data projected in this cluster and consequently they are not detected and are regarded as (false negative). The degradation of $\tau_{2}$ from $0.03 \rightarrow 0.02$, gave the birth of two maps (children) from two clusters (18 and 68) of the initial map. The first map (child) contains 90 clusters and the other contains 12 clusters (figure 2). This hierarchical extension permitted the isolation the attacks vectors of scenario 9 into one cluster classified as attack in the new map. Consequently the detection rate of attacks is increased to $96.4 \%$.

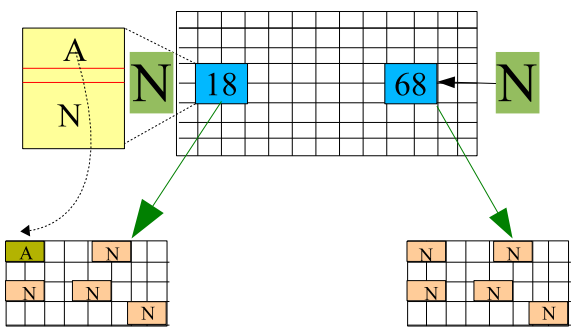

Fig. 2. Hierarchical expansion of the map obtained by decreasing the value of $\tau_{2}$ from $0.03 \rightarrow 0.02$.

In the same way the degradation of $\tau_{1}$ from $0.02 \rightarrow 0.01$ caused the addition of 3 new maps (children) in the second level. These 3 new maps are projected starting from the cluster 130 which is classified as "attacks" and which contains $3.33 \%$ of the "normal" data hidden between the vectors "attacks" of the scenario 10 projected in this cluster (see figure 3 ). This projection made it possible to separate the normal data from the attack's data and to distribute them on a great number of new clusters classified all as "normals", and to distribute the vectors of new scenarios 10 into two clusters classified as "attacks". This way of expansion or refinement decreased the "positive false" of $3.38 \%$ (see table IV).

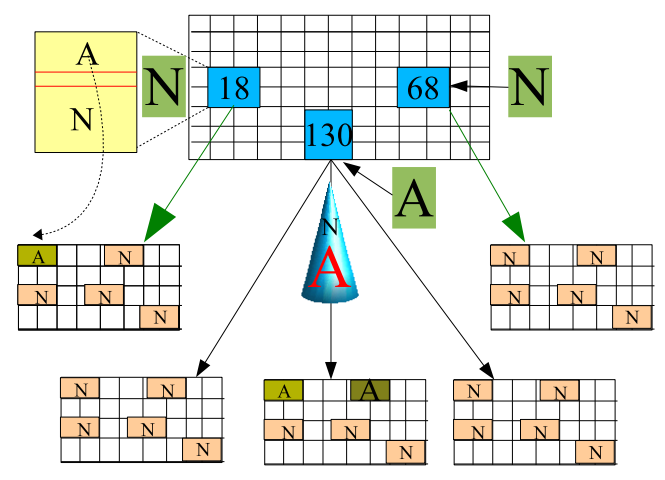

Fig. 3. Hierarchical expansion of the map obtained by decreasing the value of $\tau_{2}$ from $0.02 \rightarrow 0.01$.
TABLE VI

THE PROJECTION OF ATTACK SCENARIOS WITH THE TOP(1) CARACHTERISTIC OF EACH CLUSTER.

\begin{tabular}{|c|c|c|c|}
\hline Scnario & $\begin{array}{l}\text { Type of Sce- } \\
\text { nario }\end{array}$ & Cluster & TOP(1) \\
\hline 1 & $\begin{array}{l}\text { access page } \\
\text { denied }\end{array}$ & 121 & $\begin{array}{l}\text { Attack-responses } 403 \\
\text { forbidden }\end{array}$ \\
\hline 3 & $\begin{array}{l}\text { brute force } \\
\text { POP3 }\end{array}$ & 123 & $\begin{array}{l}\text { Incorrect Password } \\
\text { POP }\end{array}$ \\
\hline 5 & $\begin{array}{l}\text { brute force } \\
\text { FTP }\end{array}$ & 27 & Access FTP test \\
\hline 8 & $\begin{array}{l}\text { vulnerability } \\
\text { scanner }\end{array}$ & 122 & $\begin{array}{l}\text { WEB-IIS _mem_bin } \\
\text { access }\end{array}$ \\
\hline 9 & $\begin{array}{l}\text { brute force } \\
\text { FTP }\end{array}$ & 28 & Access FTP admin \\
\hline 10 & $\begin{array}{l}\text { SNMP } \\
\text { attack }\end{array}$ & 130 & SNMP request tcp ${ }^{1}$ \\
\hline 11 & $\begin{array}{l}\text { brute force } \\
\text { FTP }\end{array}$ & 27 & Access FTP test \\
\hline 14 & $\begin{array}{l}\text { brute force } \\
\text { POP3 }\end{array}$ & 114 & Incorrect User POP3 \\
\hline
\end{tabular}

\section{Qualitative Analysis}

The qualitative analysis makes it possible to measure the quality of the clusters obtained during the phase of clustering. As we mentioned in the section $\mathrm{V}$, each cluster is caracterized by the first top 5 variables of its codebook vector. The table VI show the caracteristics of the some clusters and the scenarios projected in it.

The scenarios 5 and 11 are two brute force attacks against FTP server. They are projected in the cluster 27. As we can see in the table, the top(1) carachteristics of this cluster is "Access FTP test" which is sign of this type of attacks. Another example is the scenario 10 which is a SNMP attack. This scenario is projected in the cluster 130 of which the top(1) is "SNMP request tcp", and so on. As a result, 90\% of the scenarios are projected in clusers of which the top(1) characteristic is sign of these scenarios and $100 \%$ of scenarios obtained top(3).

\section{CONCLUSION}

The major advantages of the GHSOM model over the standard SOM are the following. First, the overall training time is reduced since only a necessary number of units are developed to organize the data at a certain level of detail. Second, the GHSOM uncovers the hierarchical structure of the data, allowing the user to understand and analyse a large amount of data in an exploratory way. Each SOM array in the hierarchy explains a particular set of characteristics of data. This makes the GHSOM analysis an excellent tool for feature extraction and classification. Third, the size of the SOM array does not have to be specified subjectively before hand.

In this work, we presented the application of the GHSOM for filtering the alarms issued by a network intrusion detection system. The results obtained demonstrate that GHSOM is very suitable to this kind of problems and can overpass SOM. The 
table VII presents a summuray of the best results obtained by SOM and GHSOM.

TABLE VII

THE RESULTS OBTAINED BY GHSOM AND SOM.

\begin{tabular}{lccccc}
\hline \hline Model & DR & FP & Top(1) & Top(3) & Top(5) \\
\hline GHSOM & $96 \%$ & $4.7 \%$ & $90 \%$ & $100 \%$ & $100 \%$ \\
SOM & $70 \%$ & $15 \%$ & $33 \%$ & $83 \%$ & $83 \%$ \\
\hline \hline
\end{tabular}

\section{REFERENCES}

[1] J. Allen, A. Christie, W. Fithen, J. McHugh, J. Pickel, and E. Stoner, "State of the practice of intrusion detection technologies," Carnegie Mellon University, Tech. Rep., 2000.

[2] R. Bace, Intrusion Detection. Macmillan Technical Publishing, 2000.

[3] H. Debar, M. Dacier, and A. Wespi, "A revised taxonomy for intrusion detection systems," Annales des Télécommunications, Tech. Rep. 55(78), 2000.

[4] W. Lee, "Applying data mining to intrusion detection: the quest for automation, efficiency, and credibility," SIGKDD Explor. Newsl., vol. 4, no. 2, pp. 35-42, 2002.

[5] T. Kohonen, Self-Organizing Maps, 3rd ed., ser. Series in Information Sciences. Berlin: Springer, 2001, vol. 30.

[6] F. V. Jensen, An introduction to Bayesian Networks. London, United Kingtom: Taylor and Francis, 1996.

[7] D. M. Michael Dittenbach, Andreas Rauber, "Uncovering hierarchical structure in data using the growing hierarchical self-organizing map," Neurocomputing, vol. 48, no. 1-4, pp. 199-216, October 2002.
[8] J. Zimmermann, L. M, and C. Bidan, "Introducing reference control for intrusion detection at the os level," in Proceedings of the 5th International Symposium on the Recent Advances in Intrusion Detection, I. Network and D. S. S. S. N. 2000), Eds. Springer Verlag, 2002, pp. $157-170$.

[9] D. Zamboni, "Using internal sensors for computer intrusion detection," Ph.D. dissertation, Purdue university, 2001.

[10] M. Almegren, H. Debar, and M. Dacier, "A lightweight tool for detecting web server attacks," in Network and Distributed System Security Symposium (NDSS 2000), 2000, pp. 157-170.

[11] R. Sekar, Y. Guang, S. Verma, and T. Shanbhag, "A high performance network intrusion detection system." in 6th ACM Conference on Computer and Communications Security, 1999, pp. 8-17.

[12] F. Cuppens, "Managing alerts in a multi-intrusion detection environment," in In 17th Annual Computer Security Applications Conference (ACSAC), 2001, pp. 22-31.

[13] H. Debar and A. Wespi, "Aggregation and correlation of intrusion alerts." in In 4th Workshop on Recent Advances in Intrusion Detection (RAID 2001), L. S. Verlag, Ed., Berlin, 2001, pp. 85-103.

[14] S. Staniford, J. Hoagland, and J. McAlernay, "Practical automated detection of stealthy portscans," in ACM Computer and Communications Security IDS Workshop, 2000, pp. 1-7.

[15] K. Julish and M. Dacier, "Mining intrusion detection alarms for actionable knowledge," in 8th ACM International Conference on Knowledge Discovery and Data Mining, 2002, pp. 366-375.

[16] B. Morin and H. Debar, "Correlation of intrusion symptoms: An application of chronicles." in RAID, 2003, pp. 94-112.

[17] P. Ning, Y. Cui, and D. Reeves, "Constructing attack scenarios through correlation of intrusion alerts." in In 9th ACM Conference on Computer and Communications Security., November 2002.

[18] F. Cuppens and A. Mige, "Alert correlation in a cooperative intrusion detection framework." in In proceedings of the 2002 IEEE Symposium on Security and Privacy, Oakland, CA, May 2002, pp. 202-215.

[19] S. Cheung, U. Lindqvist, and M. W. Fong, "Modeling multistep cyber attacks for scenario recognition," in In proceedings of the third DARPA Information Survivability Conference and Exposition (DISCEX), Washington, D.C., April 2003.

[20] P. Ning, D. Xu, C. Healey, and R. Amant, "Building attack scenarios through integration of complementary alert correlation methods." in In proceedings of the 11th Annual Network and Distributed System Security Symposium (NDSS'04)., San Diego, CA, February 2004.

[21] M. Roesch, "Snort - lightweight intrusion detection for networks," in Proceedings of Thirteenth Systems Administration Conference (LISA '99), 1999, pp. 229-238.

[22] G. W. Milligan and M. C. Cooper, "An examination of procedures for determining the number of clusters in a data set," Psychometrika, 1985.

[23] P. Leray and O. Francois, "Réseaux bayésiens pour la classification méthodologie et illustration dans le cadre du diagnostic médical," Revue d'Intelligence Artificielle, vol. 18/2004, pp. 169-193, 2004.

[24] J. Pearl, Probabilistic Reasoning in Intelligent Systems: Networks of Plausible Inference. Morgan Kaufmann, 1988. 